COELIAC DISEASE

\title{
Malignant lymphomas in coeliac disease: evidence of increased risks for lymphoma types other than enteropathy- type $T$ cell lymphoma
}

\author{
K E Smedby, M Åkerman, H Hildebrand, B Glimelius, A Ekbom, J Askling
}

Gut 2005;54:54-59. doi: 10.1136/gut.2003.032094

See end of article for authors' affiliations

\section{Correspondence to:}

Dr K E Smedby

Department of Medical

Epidemiology and

Biostatistics, Karolinska

Institutet, Box 281, SE-171

77 Stockholm, Sweden;

karin.ekstrom@meb.ki.se

Revised version received

14 April 2004

Accepted for publication

25 April 2004
Background: Numerous studies have reported on the association between coeliac disease and the otherwise uncommon enteropathy-type T cell lymphoma (ETTL). A systematic risk assessment of more prevalent lymphoma entities, such as B cell and non-intestinal lymphomas, in coeliac disease has not been performed.

Aims: In light of the increasing number of patients diagnosed with coeliac disease and the unknown aetiology of malignant lymphomas, we aimed to estimate the distribution and risk of lymphoma subtypes in coeliac disease.

Methods: We reviewed and reclassified 56 cases of incident malignant lymphomas occurring in a Swedish population based cohort of 11650 patients hospitalised with coeliac disease. The observed numbers of lymphoma subtypes were compared with those expected in the Swedish population.

Results: The majority ( $n=32,57 \%$ ) of lymphomas in the cohort were not intestinal T cell lymphomas. Significantly increased risks were observed for B cell non-Hodgkin lymphoma (NHL) (standardised incidence ratio (SIR) 2.2 (95\% confidence interval (CI) 1.2-3.6); 11 non-intestinal and five intestinal) and for lymphomas of non-intestinal origin (SIR 3.6 (95\% Cl 2.3-5.2), 11 B and 14 T cell). Furthermore, $44 \%$ of patients with B cell NHL had a history of other autoimmune/inflammatory diseases. The relative risks for $\mathrm{T}$ cell NHL (SIR 51 (95\% Cl 35-68); $n=37$ ) and for primary gastrointestinal lymphomas (SIR 24 (95\% Cl 1634); five $B$ and 25 T cell) were markedly increased, as anticipated.

Conclusion: Most lymphomas complicating coeliac disease are indeed related to the disease and are not of the ETTL-type. There was a remarkable aggregation of autoimmune/inflammatory disorders, female sex, coeliac disease, and B cell lymphoma.
S ince first described in 1962, ${ }^{1}$ the association between coeliac disease and malignant lymphoma has been the focus of numerous reports. In particular, case reports ${ }^{1-3}$ and case series ${ }^{4-9}$ have linked gluten sensitive villous atrophy to a characteristic lymphoma of the small intestine, now referred to as enteropathy associated $\mathrm{T}$ cell lymphoma, or enteropathy-type T cell lymphoma (ETTL) according to the most recent lymphoma classification (WHO). ${ }^{10}$ Whether coeliac disease is also associated with an increased risk of more prevalent lymphoma types, such as non-intestinal $\mathrm{T}$ cell lymphomas or B cell lymphomas, is not clear. Most previous studies of cancer in coeliac disease are limited by small numbers ${ }^{4}{ }^{7}$ and are neither population based ${ }^{4-9}$ nor based on prospectively identified cohorts of individuals with coeliac disease $^{569}$ and are therefore subject to poor precision and risk of selection and reporting bias, both acting in favour of gastrointestinal $\mathrm{T}$ cell lymphomas. However, in the most unbiased study to date, ${ }^{11}$ six lymphomas were described in individuals with coeliac disease, of which only one case was an ETTL, indicating the possibility of a more general increase in lymphoma risk in coeliac disease. This possibility is potentially important as coeliac disease is more common than previously thought (affecting approximately 1 in every 100-200 persons in both Europe ${ }^{12}$ and North America ${ }^{13}$ ) and the aetiology of malignant lymphomas is still largely unknown ${ }^{14}$. If patients with coeliac disease are at increased risk of malignant lymphomas other than ETTL, this may have implications for the clinical surveillance of these patients as well as for our understanding of the aetiology of malignant lymphomas.
In a recent population based, prospective, cohort study of 11650 individuals hospitalised with coeliac disease in Sweden in 1964-1995, we observed a sixfold increase in lymphoma risk overall ${ }^{15}$ and a tendency for the relative risk of malignant lymphomas to decrease over successive calendar periods. Because of the lack of systematic and unbiased characterisation of malignant lymphomas arising in coeliac disease, we retrieved tumour specimens and medical files for cases with malignant lymphomas that occurred during the follow up of our cohort. The final analyses included 56 patients with verified diagnoses of coeliac disease and malignant lymphoma.

\section{PATIENTS AND METHODS}

Underlying coeliac disease cohort and identification of incident lymphomas

The cohort of individuals with coeliac disease has been described previously. ${ }^{15}$ In brief, we used the Swedish Hospital Discharge Register that contains individual based information on inpatient care countywise since 1964 and nationwide since 1987. ${ }^{16}$ For every hospital discharge, information on diagnoses and surgical procedures are recorded according to the International Classification of Diseases (ICD). The cohort consisted of all patients discharged with a diagnosis of coeliac disease at least once between l January 1964 and 31

Abbreviations: ETTL, enteropathy-type T cell lymphoma; HL, Hodgkin lymphoma; ICD, International Classification of Diseases; MALT, mucosa associated lymphoid tissue; NHL, non-Hodgkin lymphoma; SIR, standardised incidence ratio; UNS, unspecified; OR, odds ratio 
December $1995(\mathrm{n}=11605)$. ICD codes used were 286.00 (ICD 7), 269.00, 269.98 (ICD 8), and 579A (ICD 9). Using the national registration numbers, the cohort was linked to the Swedish Cancer Registry (estimated coverage $99 \%{ }^{17}$ ). Seventy seven patients with non-Hodgkin lymphoma (NHL) (ICD 7: 200, 202) or Hodgkin lymphoma (HL) (ICD 7: 201) were recorded, including cases diagnosed at autopsy $(n=7)$. Using information from the registers, the medical files of each lymphoma case were collected. Of the 77 patients registered with a diagnosis of coeliac disease preceding that of malignant lymphoma, 66 cases were confirmed as having coeliac disease on review of the medical records. We were able to reliably review tumour material in 56 of these (see below).

\section{Review of medical files}

Patients who were subsequently diagnosed as having a lymphoma were diagnosed as coeliacs between 1966 and 1994, with more being diagnosed in the 1970s $(n=24)$ than in other decades. Lymphomas were detected between 1972 and 1995, mostly during the 1980s $(n=32)$. The following information was obtained for coeliac disease: date of diagnosis, type and duration of preceding symptoms, histopathological evaluation of duodenal biopsy, performance of antigliadin antibody tests, and clinical response to a gluten free diet; and for malignant lymphoma: date of diagnosis, site/sites of presentation, and clinical stage according to Ann Arbor. ${ }^{18}$ On review of the medical records, eight patients did not have coeliac disease but mimicking disorders (tropical sprue, pancreatic exocrine insufficiency $(\mathrm{n}=2)$, lactose intolerance $(\mathrm{n}=2)$, hypogammaglobulinaemia, intestinal lymphoma without notation of coeliac disease, and transient gluten intolerance) which had led to erroneous registration. In another three patients, coeliac disease was suspected but not confirmed with a sufficient level of certainty due to atypical symptoms and/or lack of duodenal biopsies. In all but two of the remaining 66 patients, the diagnosis of coeliac disease had been histologically confirmed by duodenal biopsy. In two individuals without biopsies, the diagnosis was based on the combination of typical symptoms, prompt effect of a gluten free diet, and relapse of symptoms on gluten provocation.

\section{Review of tumour specimens}

Among the 66 individuals with confirmed coeliac disease, original tumour slides and paraffin embedded tissue blocks for the lymphoma were retrieved for all but eight patients

Table 1 Clinical picture at diagnosis of coeliac disease and results of diagnostic procedures for the 56 reviewed patients who subsequently developed malignant lymphomas

\begin{tabular}{lc}
\hline & $\begin{array}{c}\text { No of patients } \\
(\%)(\mathbf{n}=56)\end{array}$ \\
\hline $\begin{array}{l}\text { Symptoms and signs at coeliac disease diagnosis } \\
\text { Diarrhoea }\end{array}$ & $40(71)$ \\
Weight loss & $28(50)$ \\
Abdominal discomfort & $12(21)$ \\
Malabsorption (iron, folate, cobalamin, xylos, etc) & $36(64)$ \\
Results of diagnostic tests & $54(96)$ \\
Villous atrophy in duodenal biopsy & $8(14)^{*}$ \\
High antigliadin antibody titres & $45(80) \dagger$ \\
Symptomatic response to gluten free diet & $16(29) \ddagger$ \\
Histological response to gluten free diet & \\
\hline *An antigliadin antibody test was carried out in only eight patients. \\
$\begin{array}{l}\text { †One case was not treated with a gluten free diet, and for one case } \\
\text { information on diet effects was missing }\end{array}$ \\
$\begin{array}{l}\ddagger \text { A second biopsy to evaluate histological recovery was taken in } 20(36 \%) \\
\text { cases. }\end{array}$
\end{tabular}

(seven of whom were diagnosed at a hospital that chose not to cooperate). Furthermore, the condition of the tumour material precluded a reliable review for two patients (both diagnosed at autopsy). Thus a histopathological review and reclassification was performed in 56 patients $(56 / 66,85 \%)$. This process included re-evaluation of original slides in order to select appropriate blocks with preserved tumour areas. New sections were then cut from the selected blocks for routine and immunostainings. The new slides were stained with haematoxylin-eosin, and according to May-GrünwaldGiemsa and a panel of antibodies for lymphoma subtyping. All antibodies were commercially available and proven to function on formalin fixed paraffin embedded specimens. Microwave processing was used for antigen regeneration. All lymphomas were stained with a panel consisting of the B cell antibody CD20 and T cell antibodies CD3 and CD45 ${ }^{\mathrm{RO}}$. For further analyses and subtyping, antibodies were used when appropriate (based on the WHO classification criteria) from the following panel: CD2, CD5, CD10, CD15, CD23, CD30, kappa, lambda, Mib-1 (Ki-67), granzym, perforin, cyclin D1, TIA-1, Cam5.2, MNF116, and myeloperoxidase. The new slides were reviewed by an experienced haematopathologist (MÅ) who, blinded to all clinical data, reclassified the malignant lymphomas according to the WHO classification. ${ }^{10}$ In brief, all B cell lymphomas expressed CD20. In the mucosa associated lymphoid tissue (MALT) lymphomas there were evident lymphoepithelial lesions and in the mantle cell lymphomas the nuclei stained for cyclin D1. All $\mathrm{T}$ cell lymphomas were negative for CD20 but expressed either CD3 or $\mathrm{CD} 45^{\mathrm{RO}}$, or both. ETTLs showed a variable cellular and nuclear morphology with relatively monomorphic medium sized and large cells in most cases. In a few ETTL cases there was a dominance of a pleomorphic large cell population which stained focally for CD30 but not for EMA. All anaplastic large cell lymphomas expressed CD30 in almost all lymphoma cells and EMA in varying proportions of the lymphoma cell population.

\section{Statistical analysis}

We assessed the relative risks of malignant lymphomas according to phenotype and location, using the standardised incidence ratio (SIR). SIR was calculated by dividing the observed number of lymphomas in each category with that expected. Expected numbers were calculated as the product of sex, age, and calendar period specific person years of follow up in the underlying cohort and the corresponding incidence in the general population. As national rates are only available for NHL and HL, rates for T and B cell NHL as well as rates for primary gastrointestinal NHL and nonintestinal NHL were estimated using information from previous studies on their respective proportions ${ }^{11}{ }^{19-22}$ (personal communication, ongoing Swedish-Danish lymphoma study). Ninety five per cent confidence intervals (95\% CI) were calculated assuming a Poisson distribution for the observed cases. ${ }^{23} \mathrm{P}$ values for trends over calendar periods were tested using Poisson regression and all tests for statistical significance were two sided.

\section{RESULTS}

\section{Patient characteristics}

An overview of the most common symptoms and results of diagnostic tests for the 56 reviewed patients is presented in table 1 . Most patients $(\mathrm{n}=45,80 \%)$ were multisymptomatic at diagnosis of coeliac disease and the vast majority $(n=47$, $84 \%$ ) responded symptomatically and/or histologically to gluten free diet therapy. For the 47 responding patients, the median time from coeliac disease remission to diagnosis of lymphoma (three years) was approximately the same as from diagnosis of coeliac disease to that of lymphoma for all cases. 
Table 2 Baseline characteristics of 55 cases with coeliac disease and non-Hodgkin lymphoma (NHL), including 16 cases of $\mathrm{B}$ cell NHL, 37 cases of T cell NHL, and two cases with NHL unspecified

\begin{tabular}{llcc}
\hline & All & NHL B cell & T cell \\
\hline Sex (M/F) & $26 / 29$ & $4 / 12$ & $21 / 16$ \\
Age at coeliac disease diagnosis (y) & $59(15-75)$ & $60(32-73)$ & $59(15-75)$ \\
Age at lymphoma diagnosis (y) & $64(21-79)$ & $65(40-79)$ & $63(21-76)$ \\
$\begin{array}{l}\text { Duration of coeliac symptoms at diagnosis of } \\
\quad \text { coeliac disease (y) }\end{array}$ & $7(0.3-70)$ & $4(1-70)$ & $8(0.3-60)$ \\
$\begin{array}{l}\text { Interval between diagnosis of coeliac disease and } \\
\quad \text { lymphoma (y) }\end{array}$ & $3(0-22)$ & $3(0.1-22)$ & $3(0-15)$ \\
No of non-intestinal/intestinal cases & $25 / 30$ & $11 / 5$ & $14 / 23$ \\
\hline Values are median (range). & & & \\
\hline
\end{tabular}

At the time of diagnosis of the malignant lymphoma, all but one patient were on dietary treatment. On histopathological review, 55 of 56 cases were classified as NHL and one case as HL. Baseline characteristics of the patients with coeliac disease and incident NHL are shown in table 2. Thirty of 55 NHL cases $(55 \%)$ were of primary gastrointestinal origin. Twenty five cases $(45 \%)$ had no evidence of gastrointestinal involvement on complete clinical and radiological routine examination, and were hence regarded as non-intestinal. Compared with the expected numbers of lymphomas in the cohort, the observed numbers corresponded to a 6-7-fold increased risk of NHL overall (SIR 6.6 (95\% CI 5.0-8.6)), an unaltered risk of HL (SIR 1.0 (95\% CI 0.02-5.6)), a 24-fold increased risk of primary gastrointestinal NHL regardless of immune phenotype (SIR 24 (95\% CI 16-34)), and a more than threefold risk of non-intestinal NHL (SIR 3.6 (95\% CI 2.3-5.2)) (table 3). Female sex was more common among $\mathrm{B}$ cell than $\mathrm{T}$ cell NHL $(75 \% \quad v \quad 43 \%)$ and also among non-intestinal lymphomas $(67 \%$ v $42 \%$ among primary gastrointestinal NHL). Otherwise there were no significant differences in the baseline patient characteristics according to immune phenotype (table 2) or location (data not shown). Patients with confirmed coeliac disease whose tumour material could not be reviewed $(n=10)$ did not differ markedly from the reviewed cases at baseline.

\section{B cell lymphomas}

Sixteen NHL cases were of B cell lineage, corresponding to a more than doubled risk (SIR 2.2 (95\% CI 1.3-3.6)) (table 3). If the first year of follow up was excluded, the relative risk remained doubled (SIR 2.0 (95\% CI 1.1-3.4), n=13). Five cases $(31 \%)$ were of primary gastrointestinal origin (four small intestine and one gastric ventricle). Of the 11 cases with non-intestinal B cell NHL (69\%), five had nodal, one extranodal, and four both nodal and extranodal disease

Table 3 Standardised incidence ratios (SIRs) for malignant lymphomas associated with coeliac disease according to subtype (non-Hodgkin lymphoma (NHL) of B or T cell type or unspecified (UNS), Hodgkin lymphoma $(\mathrm{HL}))$ and site of presentation (intestinal/non-intestinal)

\begin{tabular}{lccc}
\hline & \multicolumn{3}{l}{ No of cases } \\
\cline { 2 - 4 } & Obs & $\operatorname{Exp}$ & SIR $(95 \% \mathrm{CI})$ \\
\hline $\mathrm{NHL}$ & 55 & 8.3 & $6.6(5.0-8.6)$ \\
$\mathrm{HL}$ & 1 & 1.0 & $1.0(0.02-5.6)$ \\
$\mathrm{NHL}$ & 16 & 7.3 & $2.2(1.3-3.6)$ \\
B cell & & & \\
T cell & 37 & 0.8 & $51(35-68)$ \\
UNS & 2 & 0.3 & $8.1(1.0-29)$ \\
Intestinal & 30 & 1.2 & $24(16-35)$ \\
Non-intestinal & 25 & 7.1 & $3.6(2.3-5.2)$ \\
\hline \multicolumn{2}{l}{$95 \% \mathrm{Cl}, 95 \%$ confidence interval. } & & \\
\hline
\end{tabular}

spread (table 4). Extranodal non-intestinal sites included bone marrow, skin, liver, pancreas, and lung. Observed numbers of both intestinal and non-intestinal B cell NHL types, respectively, were increased compared with expected numbers of intestinal versus non-intestinal NHL overall (non-intestinal: 11 observed cases $v$ maximum seven expected (non-significant); intestinal: five observed cases $v$ maximum 1.2 expected; $\mathrm{p}<0.05$ ). The classification of histological subtypes revealed a mix of indolent and aggressive lymphomas (table 5 ). The most common subtype was diffuse large B cell lymphoma $(n=7)$. B cell lymphomas occurring in the small intestine were classified as either MALT lymphoma $(n=2)$ or diffuse large B cell lymphoma $(n=2)$. No clear temporal trend was observed (table 6$)$. The majority of cases $(75 \%)$ had limited disease (stage I-II) at diagnosis (table 4). The mean survival time among B cell NHL cases was six years (median 4 years (range $0-14)$ ), and five year survival was $44 \%$. Seven patients (six women and one man) were previously diagnosed with other autoimmune or infectious disorders at the time of coeliac disease diagnosis: five had thyroid disorders with either hyper- or hypothyroid function (of which one also had a history of sarcoidosis) and two had a history of tuberculosis.

\section{T cell lymphomas}

With 37 observed cases, the relative risk for T cell lymphomas was increased 50-fold (SIR 51 (95\% CI 35-68)) (table 3). Exclusion of the first year of follow up had little impact on the relative risk (data not shown). Twenty three of 37 cases $(62 \%)$ were of primary gastrointestinal origin (22 small intestine and one colon) and $14(38 \%)$ presented in nonintestinal sites with either nodal $(n=4)$, extranodal $(n=3)$, or combined $(n=7)$ organ involvement (table 4$)$. Extranodal non-intestinal sites included skin, pleura, skeleton, and liver. Both intestinal and non-intestinal $\mathrm{T}$ cell NHLs were substantially increased compared with expected numbers of intestinal NHL overall and T cell NHL overall (intestinal: 23 observed cases $v$ maximum 1.2 expected, $\mathrm{p}<0.01$; nonintestinal: 14 observed cases $v$ maximum 0.8 expected, $\mathrm{p}<0.01)$. Nineteen $(51 \%)$ of the $\mathrm{T}$ cell lymphomas were of the ETTL subtype (all with small intestinal location). Six cases were classified as anaplastic large cell lymphomas (all non-intestinal) and 10 cases as unspecified peripheral $\mathrm{T}$ cell lymphomas (including four with gastrointestinal involvement) (table 5). Over successive calendar periods, the relative risk of $\mathrm{T}$ cell lymphomas decreased ( $\mathrm{p}$ for linear trend $=0.004$, table 6 ). The decrease applied to both intestinal and non-intestinal $\mathrm{T}$ cell lymphomas (data not shown). The majority of the T cell lymphoma patients (65\%) had limited spread of disease at diagnosis (stage I or II) (table 4). Mean survival time after diagnosis was four years (median 0.5 years (range 0-30)) and five year survival was $25 \%$. The group of patients with ETTL had a mean survival of 5.3 years and $32 \%$ lived five years after diagnosis. At the time 
Table 4 Number (\%) of patients in major subgroups of non-Hodgkin lymphoma (NHL) with nodal, extranodal, or combined organ involvement, and clinical stage according to Ann Arbor $^{18}$

\begin{tabular}{|c|c|c|c|c|}
\hline & $\begin{array}{l}\text { All } \\
(n=55)^{*}\end{array}$ & $\begin{array}{l}\text { NHL B cell } \\
(n=16)\end{array}$ & $\begin{array}{l}\text { T cell } \\
(n=37)\end{array}$ & $\begin{array}{l}\text { Primary } \\
\text { gastrointestinal } \\
(n=30)\end{array}$ \\
\hline Nodal involvement only & $9(16 \%)$ & $5(31 \%)$ & $4(11 \%)$ & 0 \\
\hline Extranodal involvement only & $19(35 \%)$ & 3 (19\%) & $16(43 \%)$ & $15(50 \%)$ \\
\hline Nodal and extranodal involvement & $27(49 \%)$ & $8(50 \%)$ & $17(46 \%)$ & $15(50 \%)$ \\
\hline \multicolumn{5}{|l|}{ Ann Arbor } \\
\hline I & 19 (34\%) & $5(31 \%)$ & $14(38 \%)$ & $15(50 \%)$ \\
\hline ॥ & $17(31 \%)$ & $7(44 \%)$ & $10(27 \%)$ & $7(23 \%)$ \\
\hline III & $7(13 \%)$ & $2(12 \%)$ & $4(11 \%)$ & $1(3 \%)$ \\
\hline IV & $12(22 \%)$ & $2(12 \%)$ & $9(24 \%)$ & $7(23 \%)$ \\
\hline
\end{tabular}

of coeliac disease diagnosis, one patient was previously diagnosed with Sjögren syndrome, one with dermatitis herpetiformis, and one patient had a history of hyperthyroidism.

\section{DISCUSSION}

Our study, which is the first large scale population based assessment of lymphoma characteristics in coeliac disease, has demonstrated that the association between coeliac disease and malignant lymphomas is not confined to ETTL but includes other types of $\mathrm{T}$ cell NHLs and, more importantly, B cell NHLs that constitute the vast majority of malignant lymphomas in the general population. Indeed, ETTL cases comprised only one third of NHL cases and half of all $\mathrm{T}$ cell lymphomas in our study, values markedly lower than previously reported. ${ }^{4-8}{ }^{24}$ Furthermore, with respect to location, our study indicated that the association was not confined to primary gastrointestinal lymphomas but also included malignant lymphomas outside of the gastrointestinal tract of both the $\mathrm{B}$ and $\mathrm{T}$ cell phenotype. Few previous studies have classified coeliac disease associated malignant lymphomas using modern immunostaining methods. ${ }^{7811}$ In one of these, 23 of 24 reviewed cases were ETTL. ${ }^{8}$ In another, 11 of 13 lymphomas were of the T cell type and eight were of intestinal origin (nodal or extranodal). ${ }^{7}$ Both studies however included lymphomas arising prior to the diagnosis of coeliac disease which may have favoured lymphoma types that would initiate investigation for potential coeliac disease (that

Table 5 Distribution of lymphoma subtypes according to the WHO classification ${ }^{10}$

\begin{tabular}{ll}
\hline & $\begin{array}{l}\text { No. of patients } \\
\text { ( } \mathbf{n}=\mathbf{5 6} \text { ) }\end{array}$ \\
\hline Lymphoma subtype & 7 \\
\hline Mature B cell neoplasms & 3 \\
Diffuse large B cell lymphoma & 2 \\
Follicular lymphoma & 2 \\
Extranodal marginal zone B cell lymphoma of MALT & 1 \\
Mantle cell lymphoma & 1 \\
Unspecified low grade B cell lymphoma & \\
Unspecified B cell lymphoma & 19 \\
Mature T cell and NK cell neoplasms & 6 \\
Enteropathy-type T cell lymphoma & 10 \\
Anaplastic large cell lymphoma & 1 \\
Peripheral T cell lymphoma, unspecified & 1 \\
Extranodal NK/T cell lymphoma, nasal type & \\
Subcutaneous panniculitis-like T cell lymphoma & 1 \\
Hodgkin lymphoma & \\
Mixed cellularity classical Hodgkin lymphoma & 1 \\
Unspecified lymphomas & 1 \\
Unspecified high grade NHL & \\
Unspecified NHL & \\
\hline MALT, mucosa associated lymphoid tissue; NK, natural killer; NHL, non- \\
Hodgkin lymphoma.
\end{tabular}

is, gastrointestinal lymphomas, particularly of the $\mathrm{T}$ cell phenotype). Older studies, mainly case series, have predominantly reported cases with gastrointestinal tumour origin. ${ }^{14-624}$ Limitations including poorly defined coeliac disease cohorts ${ }^{156}$ and case recruitment mainly from university hospitals ${ }^{4}{ }^{6}$ may have led to overrepresentation of unusual cases both with respect to coeliac disease and lymphoma. A recent Italian population based case control study of NHL (including six cases associated with coeliac disease) reported increased odds ratios (ORs) close to 20-fold for coeliac disease associated $\mathrm{T}$ cell lymphoma and primary gastrointestinal NHL, respectively, but precision was low, and ORs of B cell NHL and non-intestinal NHL were not quantified. ${ }^{11}$ A low case participation rate $(<50 \%)$ and exclusion of patients already on treatment further limited inferences from the study. Interestingly, a Swedish population based study of malignant lymphomas in association with dermatitis herpetiformis (a skin disorder of gluten intolerance closely related to coeliac disease) classified five of nine reviewed cases (a mix of prevalent and incident lymphomas) as B cell NHL. ${ }^{25}$

The strengths of our study include the comparatively large size, the prospectively defined population based cohort of patients hospitalised with coeliac disease, and the complete assessment of outcome through the population based cancer register. These features enabled us not only to characterise the internal distribution of incident lymphoma types but also to quantify relative risks compared with the general population for major lymphoma subtypes. The design also served to reduce the risk of selection and ascertainment bias (that would act to favour cases with unusual disease characteristics). To reduce the risk of detection bias, we only included individuals where the diagnosis of coeliac disease preceded that of lymphoma and, when all analyses were repeated with exclusion of the first year of follow up, the results did not change in any notable way. Another strength was the thorough validation of diagnoses of both coeliac disease and malignant lymphoma through medical files and review of tumour histology using modern techniques, minimising the risk of misclassification.

Our study was restricted to individuals ever hospitalised with a discharge diagnosis of coeliac disease. Patients under study therefore represent a subset (between 5\% and 50\% depending on age group and estimated prevalence of true coeliac disease in the background population) of all individuals with diagnosed or undiagnosed coeliac disease in Sweden during the study period. All inpatient care in Sweden is public and population based, and referrals are based on geography rather than financial situation. Therefore, restriction to hospitalised patients should not have introduced bias based on socioeconomic factors but may have limited the generalisability of the results. If patients 


\begin{tabular}{|c|c|c|c|c|c|c|c|c|c|}
\hline & \multicolumn{3}{|c|}{ All $\left(n=55^{*}\right)$} & \multicolumn{3}{|c|}{ NHL B cell $(n=16)$} & \multicolumn{3}{|c|}{$T$ cell $(n=37)$} \\
\hline & Obs & Exp & SIR $(95 \%$ CI) & Obs & Exp & $\operatorname{SIR}(95 \% \mathrm{CI})$ & Obs & Exp & $\operatorname{SIR}(95 \% \mathrm{CI})$ \\
\hline 1970-79 & 6 & 0.5 & $11(4.1-24)$ & 0 & 0.5 & - & 5 & 0.05 & $103(33-240)$ \\
\hline $1980-89$ & 31 & 3.3 & $9.3(6.3-13)$ & 9 & 2.9 & $3.1(1.4-5.8)$ & 21 & 0.3 & $70(43-107)$ \\
\hline $1990-95$ & 18 & 4.4 & $4.1(2.4-6.5)$ & 7 & 3.9 & $1.8(0.7-3.7)$ & 11 & 0.4 & $28(14-50)$ \\
\hline
\end{tabular}

*Includes 16 cases of B cell NHL, 37 cases of T cell NHL, and two cases of NHL unspecified. $95 \% \mathrm{Cl}, 95 \%$ confidence interval.

hospitalised with coeliac disease differ from those diagnosed and treated as outpatients only, our results may not be directly applicable to all individuals with coeliac disease. Yet, as the diagnostic biopsy was often taken on an inpatient basis during the first part of the study period, our lymphoma generating cohort is most likely composed of a mix of patients hospitalised either for diagnostic or therapeutic reasons and/or for concomitant medical conditions. Despite differences in design, size, and study population, our results with regard to $\mathrm{T}$ cell and gastrointestinal lymphomas are consistent with those of the recently reported population based Italian study. ${ }^{11}$

The varied pattern of reported coeliac disease associated lymphoma subtypes could be influenced (apart from differences in study size and design) by changes over time of the characteristics of prevalent and incident coeliac disease patients. We observed a decreasing trend in $\mathrm{T}$ cell lymphomas over successive calendar periods from the 1970s to the 1990s. Due to the uncertainty of the expected proportion of $\mathrm{T}$ cells during different time periods, this trend should however be interpreted with caution. Nevertheless, in a regional Swedish population based study where all incident lymphomas diagnosed from 1969 to 1987 were reclassified according to immune phenotype, ${ }^{20}$ a $\mathrm{T}$ cell proportion in the range of $8 \%$ of all NHL was seen (unpublished information), which is approximately the same as described for the 1990s (personal communication, ongoing Swedish-Danish lymphoma study). ${ }^{19}$ If the observed trend is true, it could be due to an increasing proportion of silent or mild cases of coeliac disease diagnosed in recent years with a presumably lower risk of $\mathrm{T}$ cell NHL, a possible protective effect of a gluten free diet suggested in some studies, ${ }^{26}{ }^{27}$ and/or to a temporal decrease in other determinants of $\mathrm{T}$ cell lymphoma. Also, because of the uncertainties of the expected proportions of primary gastrointestinal B cell NHL and gastrointestinal T cell NHL, we did not estimate relative risks for intestinal versus nonintestinal B cell or T cell NHL, respectively. Yet, conservative comparisons with expected numbers of intestinal NHL overall on the one hand, and non-intestinal and T cell NHL overall on the other, suggest increased risks for all four separate entities (intestinal/non-intestinal B cell NHL, intestinal/non-intestinal T cell NHL). In our study, patients with T cell lymphomas and ETTL survived slightly longer than in previous reports..$^{6-8} 28$ In comparison, a larger proportion of our patients were diagnosed in the early stages of disease (I and II) and they also most likely benefited from more modern treatment protocols.

As we were unable to histologically review all cases of malignant lymphomas occurring in the cohort (but 85\%) and only included reviewed cases in the analyses, the presented risk estimates are slightly underestimated. Furthermore, as the diagnoses of coeliac disease in the underlying cohort were not validated by examination of all of the 10000 medical files, the cohort is likely to contain a certain number of individuals erroneously registered with coeliac disease. The result (an inflation of the expected number of malignant lymphomas) would however also lead to underestimation of the relative risks across all subtypes, and would not introduce artefactual differences between subtype specific estimates. In addition, subtype specific estimates of risk for rare lymphomas (that is, $\mathrm{T}$ cell NHL) could also represent underestimations of true relative risks due to inflation of the expected number of rare lymphoma cases in the reference population by the coeliac disease patients themselves (diagnosed as well as undiagnosed). However, the conclusion remains essentially the same: the relative risk of T cell NHL in association with coeliac disease, including rare intestinal forms, is highly increased.

The biological mechanisms behind the development of ETTL in the gluten sensitive atrophic intestinal mucosa have been extensively explored. Cellier et al have suggested a transitory stage of gluten refractory disease with abnormal clones of intraepithelial T lymphocytes that may be classified as cryptic enteropathy associated $\mathrm{T}$ cell lymphoma. ${ }^{29}$ Whether the pathogenesis of non-intestinal T cell lymphomas involves the same mechanism is not known but a French group recently reported that aberrant monoclonal intraepithelial lymphocytes frequently disseminate into the blood and to other parts of the gastrointestinal tract. ${ }^{30}$ The observed association with B cell lymphomas is likely to involve other pathogenic pathways. Coeliac disease is currently considered, at least in part, to be an autoimmune disease characterised by chronic inflammation. ${ }^{31}$ A number of other autoimmune and inflammatory disorders, some of which occur in excess in individuals with coeliac disease, ${ }^{32} 33$ have been associated with an increased risk of malignant lymphomas and B cell NHL in particular (for example, rheumatoid arthritis, Sjögren syndrome, SLE, autoimmune thyroid disorders, sarcoidosis, tuberculosis). ${ }^{34-36}$ In our study, the group of patients with B cell NHL had a striking predominance of female sex and a high frequency of other autoimmune and inflammatory disorders $(44 \%$ of B cell NHL $v 5 \%$ among T cell NHL cases had a recorded history of other autoimmune diseases (predominantly thyroid disease) or tuberculosis). A history of thyroid disease could have confounded the observed association between coeliac disease and B cell NHL. However, the low range risk increases for lymphomas reported in most autoimmune or inflammatory disorders, ${ }^{35}$ together with the, although increased, still relatively low prevalence of other autoimmune disorders in coeliac disease ${ }^{32} 33$ would not be enough to create a significantly increased risk for B cell NHL. It may be that the development of intestinal B cell NHL in coeliac disease is conceptually similar to that of parotid gland MALT lymphoma in Sjögren syndrome or of gastric MALT lymphoma in the presence of Helicobacter infection, ${ }^{37}$ but also that coeliac disease becomes particularly lymphomagenic in individuals with a concomitant autoimmune condition or predisposition.

In conclusion, we have demonstrated, for the first time, that coeliac disease is associated with a far greater variety of 
malignant lymphoma types than previously shown, with a marked trend over calendar period. Indeed, non-intestinal B cell and T cell NHL together constituted the majority of coeliac disease associated malignant lymphomas in our prospectively identified population based cohort. Over the last years, coeliac disease has emerged as an in vivo model of the interplay between environmental factors (gliadin), autoimmunity, and chronic inflammation. Our results suggest that, in addition to this, coeliac disease may also be a useful model for the lymphomagenic potential associated with autoimmunity and chronic inflammation.

\section{ACKNOWLEDGEMENT}

Grant support was provided by the Swedish Cancer Society \#99 3951.

\section{Authors' affiliations}

K E Smedby, Department of Medical Epidemiology and Biostatistics, Karolinska Institutet, Stockholm, Sweden

M Åkerman, Department of Pathology, Lund University Hospital, Lund, Sweden

H Hildebrand, Department of Paediatrics, Astrid Lindgrens Children's Hospital, Stockholm, Sweden

B Glimelius, Department of Oncology, Radiology, and Clinical Immunology, Uppsala University Hospital and Department of Oncology and Pathology, Karolinska Hospital, Stockholm, Sweden

A Ekbom, Department of Medicine, Clinical Epidemiology Unit, Karolinska Hospital, Stockholm, Sweden

J Askling, Department of Medicine, Clinical Epidemiology Unit, and Department of Medicine, Rheumatology Unit, Karolinska Hospital, Stockholm, Sweden

Conflict of interest: None declared.

\section{REFERENCES}

1 Gough K, Read AE, Naish JM. Intestinal reticulosis as a complication of idiopathic steatorrhoea. Gut 1962;3:232-9.

2 Loughran TP Jr, Kadin ME, Deeg HJ. T-cell intestinal lymphoma associated with celiac sprue. Ann Intern Med 1986;104:44-7.

3 Isaacson PG, O'Connor NT, Spencer J, et al. Malignant histiocytosis of the intestine: a T-cell lymphoma. Lancet 1985;2:688-91.

4 Holmes GK, Stokes PL, Sorahan TM, et al. Coeliac disease, gluten-free diet, and malignancy. Gut 1976;17:612-9.

5 Isaacson $\mathrm{P}$, Wright $\mathrm{DH}$. Intestinal lymphoma associated with malabsorption. Lancet 1978;1:67-70.

6 Swinson CM, Slavin G, Coles EC, et al. Coeliac disease and malignancy. Lancet 1983;1:111-15.

7 Mathus-Vliegen EM, Van Halteren H, Tytgat GN. Malignant lymphoma in coeliac disease: various manifestations with distinct symptomatology and prognosis? J Intern Med 1994;236:43-9.

8 Egan L, Walsh SV, Stevens FM, et al. Celiac-associated lymphoma. A single institution experience of 30 cases in the combination chemotherapy era. J Clin Gastroenterol 1995;21:123-9.

9 Pricolo VE, Mangi AA, Aswad B, et al. Gastrointestinal malignancies in patients with celiac sprue. Am J Surg 1998;176:344-7.

10 Jaffe ES, Harris NL, Stein H, et al. Pathology and genetics of tumours of hematopoietic and lymphoid tissues. Lyon: IARC Press, 2001.
11 Catassi C, Fabiani E, Corrao G, et al. Risk of non-Hodgkin lymphoma in celiac disease. JAMA 2002;287:1413-19.

12 Maki M, Mustalahti K, Kokkonen J, et al. Prevalence of celiac disease among children in Finland. N Engl J Med 2003;348:2517-24.

13 Fasano A, Berti I, Gerarduzzi T, et al. Prevalence of celiac disease in at-risk and not-at-risk groups in the United States: a large multicenter study. Arch Intern Med 2003; 163:286-92.

14 Evans LS, Hancock BW. Non-Hodgkin lymphoma. Lancet 2003;362:139-46.

15 Askling J, Linet M, Gridley G, et al. Cancer incidence in a population-based cohort of individuals hospitalized with celiac disease or dermatitis herpetiformis. Gastroenterology 2002; 123:1428-35.

16 Swedish Hospital Discharge Register 1987-1996, quality and contents. Stockholm: Swedish National Board of Health and Welfare, Centre for Epidemiology, 1998.

17 Mattsson B, Wallgren A. Completeness of the Swedish Cancer Register. Nonnotified cancer cases recorded on death certificates in 1978. Acta Radiol Oncol 1984;23:305-13.

18 Carbone PP, Kaplan HS, Musshoff K, et al. Report of the committee on Hodgkin's disease staging classification. Cancer Res 1971;31:1860-1.

19 Carbone A, Franceschi S, Gloghini A, et al. Pathological and immunophenotypic features of adult non-Hodgkin's lymphomas by age group. Hum Pathol 1997;28:580-7.

20 Martinsson U, Glimelius B, Sundstrom C. Lymphoma incidence in a Swedish county during 1969-1987. Acta Oncol 1992;31:275-82.

21 Otter R, Bieger R, Kluin PM, et al. Primary gastrointestinal nonHodgkin's lymphoma in a population-based registry. $\mathrm{Br} J$ Cancer 1989;60:745-50

22 d'Amore F, Brincker H, Gronbaek K, et al. Non-Hodgkin's lymphoma of the gastrointestinal tract: a population-based analysis of incidence, geographic distribution, clinicopathologic presentation features, and prognosis. Danish Lymphoma Study Group. J Clin Oncol 1994;12:1673-84.

23 Breslow N, Day NE. Statistical methods in cancer research, vol II. The design and analysis of cohort studies. Lyon: IARC Sci Publ, 1987:1-404.

24 Logan RF, Rifkind EA, Turner ID, et al. Mortality in celiac disease. Gastroenterology 1989;97:265-71.

25 Sigurgeirsson B, Agnarsson BA, Lindelof B. Risk of lymphoma in patients with dermatitis herpetiformis. BMJ 1994;308:13-15.

26 Holmes GK, Prior P, Lane MR, et al. Malignancy in coeliac disease-effect of a gluten free diet. Gut 1989;30:333-8

27 Lewis HM, Renaula TL, Garioch JJ, et al. Protective effect of gluten-free diet against development of lymphoma in dermatitis herpetiformis. Br J Dermatol 1996;135:363-7.

28 Cooper BT, Holmes GK, Ferguson R, et al. Celiac disease and malignancy. Medicine (Baltimore), 1980;59:249-61.

29 Cellier C, Delabesse E, Helmer C, et al. Refractory sprue, coeliac disease, and enteropathy-associated T-cell lymphoma. French Coeliac Disease Study Group. Lancet 2000;356:203-8.

30 Verkarre V, Asnafi V, Lecomte T, et al. Refractory coeliac sprue is a diffuse gastrointestinal disease. Gut 2003;52:205-11.

31 Feighery C. Fortnightly review: coeliac disease. BMJ 1999;319:236-9.

32 Counsell CE, Taha A, Ruddell WS. Coeliac disease and autoimmune thyroid disease. Gut 1994;35:844-6.

33 Ventura A, Magazzu G, Greco L. Duration of exposure to gluten and risk for autoimmune disorders in patients with celiac disease. SIGEP Study Group for Autoimmune Disorders in Celiac Disease. Gastroenterology 1999; 117:297-303

34 Pedersen RK, Pedersen NT. Primary non-Hodgkin's lymphoma of the thyroid gland: a population based study. Histopathology 1996;28:25-32.

35 Baris D, Zahm SH. Epidemiology of lymphomas. Curr Opin Oncol 2000;12:383-94.

36 Baecklund E, Sundstrom C, Ekbom A, et al. Lymphoma subtypes in patients with rheumatoid arthritis: increased proportion of diffuse large $B$ cell lymphoma. Arthritis Rheum 2003;48:1543-50.

37 Burke JS. Are there site-specific differences among the MALT lymphomasmorphologic, clinical? Am J Clin Pathol 1999;111:S133-43. 